\title{
Lesson of the month 1: Pericardial mass and cardiac tamponade associated with Mycoplasma pneumoniae
}

\author{
Authors: Vinit Sawhney, ${ }^{\mathrm{A}}$ Oksana Maksunova, ${ }^{\mathrm{B}}$ Syed Ahsan, ${ }^{\mathrm{C}}$ Muhiddin Ozkor ${ }^{\mathrm{D}}$ and Mark Westwood ${ }^{\mathrm{E}}$
}

\section{Mycoplasma pneumoniae primarily causes respiratory tract infections. Extrapulmonary manifestations are seen in $20-25 \%$ of cases. Cardiac involvement is rarely reported. We present a unique case of a pericardial mass and cardiac tamponade associated with a Mycoplasma pneumoniae pneumonia. This required emergency pericardial fenestration. The patient improved dramatically postoperatively on antibiotics and there was no recurrence of the pericardial effusion on follow up. This case highlights the often forgotten invasive properties of a common respiratory tract pathogen and emphasises the need to consider this easily treatable entity in the differential diagnosis of idiopathic pericardial effusions.}

KEYWORDS: Mycoplasma pneumoniae, pericardial mass, cardiac tamponade

\section{Lesson}

A 40-year-old African-Caribbean woman presented to the emergency department with a 4-day history of worsening shortness of breath and chest pain. These were preceded by a 3-week history of fevers, a non-productive cough, fatigue and generalised aches. Significant medical history included childhood asthma and a 10-pack-year smoking history. She was not prescribed any regular medication.

Initial clinical examination revealed a temperature of $38.5^{\circ} \mathrm{C}$, a blood pressure of $107 / 85 \mathrm{mmHg}$, a heart rate of 110 beats/min and a respiratory rate of 20 breaths/min, with oxygen saturations of $95 \%$ on room air. Heart sounds were normal on auscultation and there was evidence of bronchial breathing on the right lung base with a few bibasal crepitations. Systemic examination did not reveal any other abnormalities. Electrocardiogram (ECG) showed sinus rhythm with a

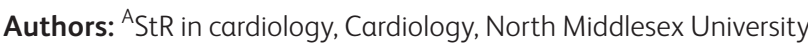
Hospital, London, UK; ${ }^{B}$ chief echo technician, Cardiology, North Middlesex University Hospital, London, UK; ' Consultant cardiologist, Cardiology, North Middlesex University Hospital, London, UK; ${ }^{\mathrm{D}}$ consultant cardiologist, Cardiology, North Middlesex University Hospital, London, UK; ${ }^{\mathrm{E}}$ Consultant cardiologist, Cardiology, North Middlesex University Hospital, London, UK ventricular rate of 102. There was a fixed anterolateral T-wave inversion with good $\mathrm{R}$-wave progression. The $\mathrm{P}-\mathrm{R}$ interval was $146 \mathrm{~ms}$ with a QTc of $427 \mathrm{~ms}$. The axis was normal with no evidence of fascicular block. The chest radiograph revealed an enlarged, globular, cardiac silhouette with right basal consolidation in the lung parenchyma. Laboratory blood tests during the course of her admission are summarised in Table 1. Mycoplasma serology was positive.

A transthoracic echocardiogram was performed. This showed a moderate pericardial effusion with a mass in the pericardial space compressing the right ventricle. Respiratory variation of Doppler velocities across the mitral and tricuspid valve suggested tamponade. Collapse of the inferior vena cava during inspiration was impaired. Left ventricular size and function were normal (Fig 1).

The patient's computed tomography (CT) scan of the chest/abdomen/pelvis confirmed a small, right-sided, pleural effusion with right basal consolidation. A large pericardial effusion was noted and there was an anterior mediastinal soft-tissue mass by the right lower lobe and pulmonary artery.

The patient was transferred to a tertiary centre for further investigations and drainage of the pericardial fluid. Subsequent cardiac magnetic resonance imaging (MRI) showed a large pericardial effusion with a mass in the pericardial space. There was also a mass posterior to the left atrium, which suggested a benign bronchial cyst.

The patient underwent a right-sided, video-assisted, thoracoscopic pericardial window using pericardial fenestration. A volume of $800 \mathrm{ml}$ pericardial fluid was drained and the pericardial mass was biopsied. Samples were sent for microbiology and histology.

The pericardial fluid was negative for acid-fast bacilli and fungal cultures. The pericardial biopsy demonstrated chronically inflamed pericardial tissue. Biopsy of the pericardial mass revealed an organising thrombus.

The patient was treated with amoxicillin and clavulanate (Augmentin), and clarithromycin. She made an excellent clinical recovery and her inflammatory markers returned to normal.

Subsequent follow-up echocardiography showed no residual pericardial effusion, normal left ventricular function and no evidence of valvular abnormalities (Fig 2).

Repeat cardiac MRI, 4 months post-pericardial drainage and biopsy, demonstrated complete resolution of the pericardial disease. The benign looking posterior mediastinal cyst 


\section{Table 1. Laboratory blood tests.}

\begin{tabular}{lll} 
Serology & On admission & Follow up \\
$\mathrm{Hb}(\mathrm{g} / \mathrm{dl})$ & 8.5 & 12 \\
$\mathrm{WCCS}\left(\mathrm{cell} / \mathrm{s} / \mathrm{mm}^{3}\right.$ ) & 13 & 8 \\
Platelets (cells $/ \mathrm{mm}^{3}$ ) & 896 & 551 \\
$\mathrm{CRP}(\mathrm{mg} / \mathrm{l})$ & 300 & 20 \\
Liver profile & Normal & Normal \\
Renal profile & Normal & Normal \\
& & \\
Mycoplasma pneumoniae IgM & 100 & \\
antibodies (U/ml) & & \\
HIV & Negative \\
Hepatitis B and C & Negative \\
Brucella and Coxiella spp. & Negative \\
ANA, ANCA, ENA, ACE & Negative \\
Pericardial fluid & Negative for AFB, fungal \\
& cultures and malignant \\
Pericardial mass biopsy & cells \\
Pericardial biopsy & Thrombus \\
\hline ACE = Chronic inflammation
\end{tabular}

$\mathrm{ACE}$ = angiotensin-converting enzyme; $\mathrm{AFB}=$ acid-fast bacillus; $\mathrm{ANA}=$ antinuclear antibody; ANCA = anti-neutrophil cytoplasmic antibody; $\mathrm{CRP}=\mathrm{C}$-reactive protein; ENA $=$ extractable nuclear antigen; WCC $=$ white cell count.

remained unchanged from the previous scan. The left and right ventricular cavity sizes and function were normal. Myocardial tissue characterisation was also normal.

\section{Discussion}

Mycoplasma pneumoniae is a common cause of atypical pneumonia in young adults. Extrapulmonary manifestations are seen in $20-25 \%$ of cases and may appear before or after, or coexist with, the pulmonary disease. ${ }^{1}$ Although extrapulmonary manifestations of M pneumoniae are well described, cardiac tamponade and pericardial masses associated with $M$ pneumonia are rarely reported.

Cardiac complications associated with M pneumoniae are relatively uncommon. The incidence of cardiac involvement

\section{Key points}

> Mycoplasma pneumoniae is a common respiratory pathogen that can cause significant cardiac complications, including pericardial mass and cardiac tamponade

> This easily treatable entity should be considered in the differential diagnosis of idiopathic pericardial effusions

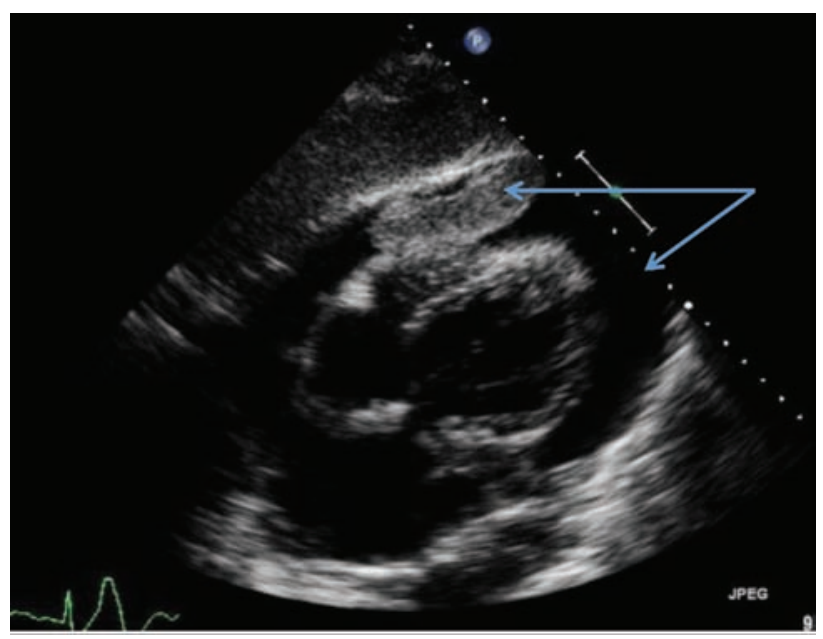

Fig 1. Transthoracic echocardiogram on admission. The pericardial mass with diastolic compression of the right ventricle is seen (shown by arrows). Respiratory variation of Doppler velocities across mitral and tricuspid valves suggests cardiac tamponade.

is estimated at $4.5 \%$ in people with serological evidence of infection. It is more common in adults and has been reported in isolated cases, manifesting largely as myopericarditis. ${ }^{2,3}$ Proposed hypotheses for M pneumonia-associated carditis include direct invasion of the myocardium by the organism (via the lymphatics, circulation or contamination from the lower respiratory tract), autoimmune mechanisms or increased tendency for intravascular coagulation. ${ }^{4}$

The present case is a unique example of a plausible association between $M$ pneumoniae and a pericardial mass and effusion, causing cardiac tamponade. It highlights the often forgotten invasive properties of a common respiratory tract pathogen, and emphasises the need to consider this easily treatable entity in the differential diagnosis of idiopathic pericardial effusions.

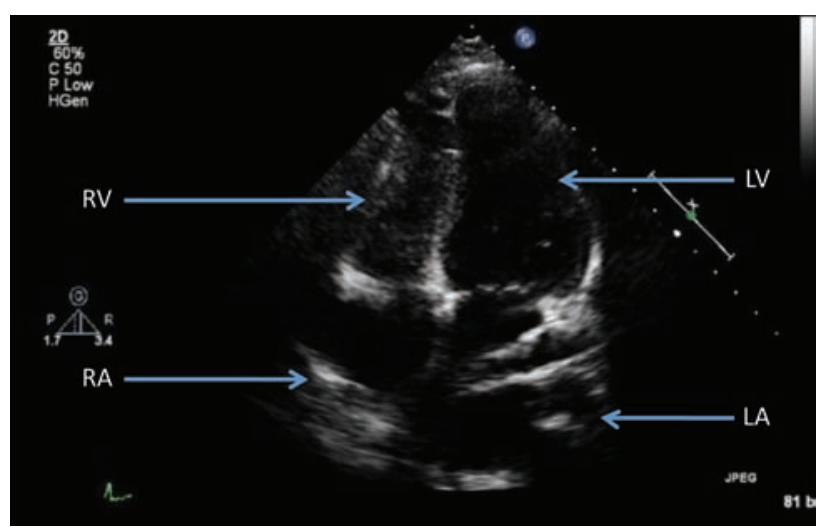

Fig 2. Normal follow-up transthoracic echocardiogram with a structurally normal heart. $L A=$ left atrium; $L V=$ left ventricle; $R A=$ right atrium; $\mathrm{RV}=$ right ventricle. 


\section{References}

1 Okoli K, Gupta A, Irani F, Kasmani R. Immune thrombocytopenia associated with Mycoplasma pneumoniae: a case report and review of literature. Blood Coagul Fibrinolysis 2009;20:595-8.

2 Izumikawa K, Izumikawa K, Takazono $\mathrm{T}$ et al. Clinical features, risk factors and treatment of fulminant Mycoplasma pneumoniae pneumonia: A review of Japanese literature. J Infect Chemother 2014;20:181-5.

3 Kenney RT, Li JS, Clyde WA Jr et al. Mycoplasmal pericarditis: evidence of invasive disease. Clin Infect Dis 1993;17:S58-62.
4 Hawkins S, Rausch CM, McCanta AC. Constrictive pericarditis secondary to infection with Mycoplasma pneumoniae. Curr Opin Pediatr 2011;23:126-9.

Address for correspondence: Dr V Sawhney, Cardiology, St Bartholomew's Hospital, 1st Floor, 60 Dominion House West, Smithfields, London EC1A 9BE.

Email: sawhneyvin@googlemail.com

\title{
Lesson of the month 2: Severe reactivation of hepatitis B after immunosuppressive chemotherapy
}

\author{
Authors: Jessica Katharine Dyson, ${ }^{A}$ Mark Hudson ${ }^{B}$ and Stuart McPherson ${ }^{C}$
}

\section{Patients with current or past hepatitis B virus (HBV) infection are at risk of viral reactivation if they receive immune-modulating treatment or chemotherapy. This can range from subclinical elevation in HBV DNA levels, to abnormal liver function tests, to severe hepatitis with liver failure and risk of death. All patients should be screened for hepatitis B with surface antigen and core antibody before receiving immunosuppression. Patients with positive hepatitis $B$ serology should be referred for specialist advice. Prophylactic antiviral treatment is recommended for patients with current/past hepatitis $B$ who receive immunosuppressive chemotherapy.}

KEYWORDS: Hepatitis B, reactivation, immunosuppression, chemotherapy, antiviral therapy.

\section{Introduction}

Patients with current or past hepatitis B virus (HBV) infection are at risk of viral reactivation if they receive immune-modulating treatment or chemotherapy. This can range from subclinical elevation in HBV DNA levels to abnormal liver function tests (LFTs), to severe hepatitis with liver failure and risk of death.

\footnotetext{
Authors: ${ }^{\text {A }}$ clinical hepatology fellow, Freeman Hospital, Newcastle upon Tyne Hospitals NHS Foundation Trust, Newcastle upon Tyne, UK; ${ }^{B}$ consultant hepatologist, Freeman Hospital, Newcastle upon Tyne Hospitals NHS Foundation Trust and Institute of Cellular Medicine, Newcastle University, Newcastle upon Tyne, UK; ${ }^{C}$ consultant hepatologist, Freeman Hospital, Newcastle upon Tyne Hospitals NHS Foundation Trust and Institute of Cellular Medicine, Newcastle University, Newcastle upon Tyne, UK
}

\section{Case report}

A 72-year-old man was noted to have submandibular gland swelling at a dental appointment. Further assessment showed widespread lymphadenopathy and excision biopsy diagnosed diffuse large B-cell lymphoma. His past medical history included pulmonary embolus.

He was planned for six cycles of R-CHOP chemotherapy (rituximab, cyclophosphamide, doxorubicin [hydroxydaunomycin], vincristine, prednisolone). Before starting treatment, hepatitis B serology was checked; he was anti-HBcAb (hepatitis B core antibody) positive, HBsAg (hepatitis B surface antigen) negative, anti-HBsAb (hepatitis B surface antibody) negative with undetectable HBV DNA $(<20 \mathrm{IU} / \mathrm{ml})$, in keeping with past HBV infection. LFTs were normal. The virology report stated: 'consistent with past HBV infection, but risk of reactivation on immunosuppression. Please ensure HBsAg and LFTs are monitored.'

After four cycles of R-CHOP (January 2013), tests for HBsAg were positive and HBV DNA was $1.2^{\prime} 10^{7} \mathrm{IU} / \mathrm{ml}$, consistent with acute $\mathrm{HBV}$ reactivation. The patient was started on tenofovir $245 \mathrm{mg}$ daily.

He completed six cycles in March 2013 and positron emission tomography computed tomography (PET-CT) confirmed good radiological response. However, in May 2013 he was admitted with jaundice and international normalised ratio (INR) $>10$. Blood tests showed significant liver dysfunction (bilirubin $105 \mu \mathrm{mol} / \mathrm{l}$, alanine aminotransferase [ALT] $988 \mathrm{U} / \mathrm{l}$, alkaline phosphatase [ALP] $317 \mathrm{U} / \mathrm{l}$ and albumin $35 \mathrm{~g} / \mathrm{l})$. HBV DNA had fallen to 5,300 IU/ml. An abdominal CT scan showed no biliary obstruction or other cause for jaundice. Tests for other hepatotrophic viruses were negative. Transjugular liver biopsy showed markedly cholestatic hepatitis (modified histology activity index [HAI] inflammatory grade $9 / 18$ and fibrosis 